\section{Correspondence \\ Paul R. Wheeler \\ p.wheeler@vla.defra.gsi.gov.uk}

Received 15 July 2008

Revised 13 August 2008

Accepted 14 August 2008

\title{
Functional analysis of a clonal deletion in an epidemic strain of Mycobacterium bovis reveals a role in lipid metabolism
}

\author{
Paul R. Wheeler, ${ }^{1}$ Roland Brosch, ${ }^{2}$ Nicholas G. Coldham, ${ }^{1}$ \\ Jacqueline K. Inwald, ${ }^{1}$ R. Glyn Hewinson ${ }^{1}$ and Stephen V. Gordon ${ }^{1,3,4,5}$ \\ ${ }^{1}$ VLA Weybridge, New Haw, Addlestone KT15 3NB, Surrey, UK \\ ${ }^{2}$ Institut Pasteur, UP Pathogénomique Mycobactérienne Intégrée, 25, Rue du Dr Roux, 75015 \\ Paris, France \\ ${ }^{3}$ School of Agriculture, Food Science and Veterinary Medicine, College of Life Sciences, University \\ College Dublin, Ireland \\ ${ }^{4}$ School of Biomolecular Science, School of Medicine and Medical Science, College of Life \\ Sciences, University College Dublin, Ireland \\ ${ }^{5}$ School of Biomedical Science, School of Medicine and Medical Science, College of Life Sciences, \\ University College Dublin, Ireland
}

Previous work on the population structure of Mycobacterium bovis strains in Great Britain has identified highly successful clones which are expanding across the country. One such clone, designated $M$. bovis type 17, differs from all other members of the Mycobacterium tuberculosis complex in having a region of deletion, termed RDbovis(d)_0173, of seven genes between Mb1963c and Mb1971. Three of these genes have functions annotated in lipid metabolism. To explore the molecular basis for the success of this clone, we examined the impact of this deletion on lipid metabolism. While type 17 isolates had similar lipid composition to other M. bovis strains, their ability to incorporate propanoate into mycolic acids was remarkably low. When expressed as a reciprocal (the ratio of incorporation of label from acetate : propanoate into mycolic acids) the ratio was higher for all three type 17 field strains tested (mean: 18.90) than the values of 7.30 to 7.61 for other field strains $(P<0.002)$ and values $<6.50$ for all other strains in the $M$. tuberculosis complex tested. The label from propanoate was diverted to pyruvate, at significantly higher levels in $M$. bovis type 17 than all other strains $(P<0.021)$. Complementation of $M$. bovis type 17 with an integrating cosmid, IE471, carrying the M. tuberculosis orthologues of Mb1963c-Mb1971 resulted in the ability of the recombinant strain to incorporate label from propanoate into mycolic acids in a manner similar to other strains. M. bovis type $17:$ : IE471 labelled pyruvate from propanoate about four times more slowly than the parent strain. Thus, RDbovis(d)_0173 results in a profound effect on carbon metabolism, providing the ability to compensate for the inactivation of the ald and pykA genes, involved in pyruvate metabolism, that is seen in $M$. bovis (but not in $M$. tuberculosis). This shift in carbon metabolism may be a factor in the extraordinary clonal expansion reported for M. bovis type 17 .

\section{INTRODUCTION}

Genomic technologies now allow us to scan pathogen genomes in remarkable depth. In the field of human tuberculosis, this has been exemplified by the work of Small and colleagues, who have used gene chip technology to identify unique event polymorphisms across a worldwide distribution of Mycobacterium tuberculosis isolates, providing unprecedented insight into the population structure of this pathogen (Gagneux et al., 2006). Furthermore, their work suggest that M. tuberculosis strains evolve to adapt to the local host population. The role of gene loss in virulence has been 
examined from a functional point of view by Wilkinson and colleagues, who showed that a gene deletion from an epidemic strain of $M$. tuberculosis conferred an altered immunomodulatory phenotype that differed from that of strains where the gene was intact (Henao-Tamayo et al., 2007). Hence, mutational events can clearly alter the phenotype of M. tuberculosis isolates in ways that may affect their success as pathogens.

While M. tuberculosis causes death and disease in humans, leading to 2 million fatalities a year worldwide, Mycobacterium bovis (which is $99.95 \%$ similar at the nucleotide level) causes financial devastation, with losses of $\$ 3$ billion a year to agriculture (Garnier et al., 2003). Molecular typing is used in tracing and surveillance of bovine tuberculosis in cattle and wildlife in the UK, and in trying to understand why the epidemic is increasing year on year - in contrast to continental Europe, where a similar test and slaughter control policy has reduced the incidence in cattle to a negligible level (Smith et al., 2006). The current means of bovine tuberculosis control in Great Britain (GB: England, Scotland and Wales) is the 'test and slaughter' strategy, whereby animals giving a positive skin reaction to a crude preparation of mycobacterial antigens are identified as infected and subsequently slaughtered. Cases of bovine tuberculosis are then confirmed by culturing the bacilli from post-mortem material. M. bovis isolates are analysed at VLA Weybridge by spaceroligonucleotide typing (spoligotyping), which is based on a polymorphic region of the genome called the direct repeat (DR) locus that is composed of multiple, identical 36 bp DR repeats interspersed by unique sequences called spacers. Isolates of $M$. bovis differ in the presence or absence of spacers and adjacent DRs, allowing a 'barcode' to be generated for each molecular type (Kamerbeek et al., 1997). Spoligotype patterns are named at VLA on the basis of a sequential numbering system; e.g. spoligotype 9 was the ninth pattern to appear, spoligotype 17 the seventeenth, etc. One notable feature of the UK epidemic is that $60 \%$ of the affected cattle are infected by just two spoligotypes of M. bovis, type 9 and the essentially clonal type 17 . Type 17 represents an emerging microepidemic as it has increased significantly compared with all other field strains of $M$. bovis (Smith et al., 2003). Furthermore, we have used Fourier-transform infrared spectroscopy (FT-IR) to measure the vibration characteristics of functional groups across replicate cultures of the major spoligotypes. Using this analysis one can measure quantitative differences in polysaccharides, proteins, lipids, etc., between samples. Strikingly, multivariate statistical analysis clustered the resultant spectra in agreement with the molecular phylogeny; for example, all type 17 strains clustered together (Winder et al., 2006). This global metabolic analysis therefore showed that strains of the same molecular type have distinct phenotypic traits.

Given that type 17 strains are a growing threat to cattle in the UK, and are increasing as a result of natural selection rather than genetic drift (Smith et al., 2003), we were interested in discovering the biochemistry that underlies the selectable phenotype of type $17 \mathrm{M}$. bovis. A major clue is that the phenotype is related to lipid biochemistry, given that type 17 has a small region of deletion, termed RDbovis(d)_0173 (Mostowy et al., 2005). This deletion affects seven genes between Mb1963c and Mb1971 ( $M$. tuberculosis $\mathrm{H} 37 \mathrm{Rv}$ designation $R v 1928 c-R v 1936)$, three of which are annotated as being involved in fatty acid degradation (Table 1). The expected substrates for the three predicted enzymes are fatty acyl-CoA for the FadEs and 2-unsaturated enoyl-CoA for the EchA. However, with $21 \mathrm{ech} A$ genes and $36 \mathrm{fadE}$ genes, each encoded enzyme may have a narrow specificity, rather than there being massive redundancy. Yet there is no clue what the substrates of these deleted genes might be, nor is it clear whether their deletion affects catabolism or anabolism. Although they are genes associated with the catabolic, $\beta$ oxidation pathway, their deletion could allow the accumulation of fatty acids and polyketides and might even influence remodelling of mycobacterial lipids with changed fatty acyl moieties. Therefore, the global metabolic labelling of lipids was analysed in strains with and without the RDbovis(d)_0173 genes.

Table 1. RDbovis(d)_0173: genes deleted in M. bovis type 17

\begin{tabular}{|lll|}
\hline M. bovis gene & $\begin{array}{c}\text { M. tuberculosis } \\
\text { orthologue }\end{array}$ & \multicolumn{1}{c|}{ Predicted product } \\
\hline$M b 1963 c$ & $R v 1928 c$ & Probable short-chain type dehydrogenase/reductase \\
$M b 1964 c$ & $R v 1929 c$ & Conserved hypothetical protein \\
$M b 1965 c$ & $R v 1930 c$ & Conserved hypothetical protein \\
$M b 1966 c$ & $R v 1931 c$ & Probable transcriptional regulatory protein \\
$M b 1967$ & $t p x$ & Probable thiol peroxidase tpx \\
$M b 1968 c$ & $F a d E 18$ & Probable acyl-CoA dehydrogenase fadE18 \\
$M b 1969 c$ & $F a d E 17$ & Probable acyl-CoA dehydrogenase fadE17 \\
$M b 1970 c$ & $E c h A 13$ & Possible enoyl-CoA hydratase echA13 (enoyl hydrase) \\
& (unsaturated acyl-CoA hydratase) (crotonase) \\
$M b 1971$ & $R v 1936$ & Possible monooxygenase \\
\end{tabular}


The outcome of the global metabolic labelling experiments reported in this study was a striking difference between the labelling of mycolic acids with propanoate between type 17 M. bovis and every other strain examined. Therefore, we focussed on how propanoate might label mycolates and alternative products. Mycolic acids are present in all actinobacteria, whether they are saprophytes, pathogens, or even those used in biotechnology such as Corynebacterium glutamicum and M. bovis BCG, which is used for the production of the vaccine against tuberculosis (Goodfellow et al., 1976). Although the biotechnology strains have a much changed metabolism to adapt to the media used for their growth (Guerin, 1980) and for production requirements (Jetten \& Sinskey, 1995), the mycolic acids remain essentially unchanged. This is not surprising since most of the mycolic acids are covalently bound, via a branched glycan, to the peptidoglycan (McNeil et al., 1990). As such, they form the inner leaf of an outer permeability membrane, complete with porins (Niederweis, 2003). While mycolates are universal to actinobacteria, loss of some classes of mycolate (Behr et al., 2000), and even more subtle structural differences in their cyclopropane ring structures, can lead to changes in the virulence of mycobacteria (Glickman et al., 2000). In the current work, no differences in structure or abundance of mycolic acid classes were detected between strains. However, it was established that mycolic acids are labelled much less efficiently with propanoate by $M$. bovis type 17 than any other $M$. bovis strain and all strains of $M$. tuberculosis tested. Thus, verification of this finding and investigation of the metabolic consequences of this differential efficiency of labelling with propanoate were the major areas we investigated after obtaining the global data.

\section{METHODS}

Strains and their culture. An objective before starting metabolic labelling experiments was to be able to grow all strains on the same medium, using the same carbon source, at the same rate. All the strains in Table 2 were grown in Sauton medium with pyruvate replacing glycerol as sole carbon source. The composition was: L-asparagine, $4 \mathrm{~g} \mathrm{l}^{-1}$; citric acid, $2 \mathrm{~g} \mathrm{l}^{-1} ; \mathrm{K}_{2} \mathrm{HPO}_{4}, 0.5 \mathrm{~g} \mathrm{l}^{-1}$; $\mathrm{MgSO}_{4} .7 \mathrm{H}_{2} \mathrm{O}, 0.5 \mathrm{~g} \mathrm{l}^{-1}$; ammonium ferric citrate, $0.05 \mathrm{~g} \mathrm{l}^{-1}$; sodium pyruvate, $4.16 \mathrm{~g} \mathrm{l}^{-1}$. The pyruvate had to be added to get any growth at all. Deionized water was used dissolve the medium constituents and the $\mathrm{pH}$ was adjusted with $\mathrm{KOH}$ to $\mathrm{pH}$ 7.2. In experiments with alternative sole carbon sources, pyruvate was omitted and either sodium propanoate at $2 \mathrm{~g} \mathrm{l}^{-1}$, or Tween 80 at $2 \mathrm{~g} \mathrm{l}^{-1}$ with BSA at $5 \mathrm{~g}$ $1^{-1}$ was included. For growth of recombinant strains that contained

Table 2. Strains and cosmids used in this study

\begin{tabular}{|c|c|c|c|c|}
\hline Cosmid or strain & SB no.* & Description & $\begin{array}{l}\text { RDbovis } \\
\text { (d)_0173† }\end{array}$ & Source or reference \\
\hline \multicolumn{5}{|l|}{ Cosmids } \\
\hline IE471 & & $\begin{array}{l}\text { pYUB412-based integrating cosmid con- } \\
\text { taining a } M \text {. tuberculosis Erdman insert } \\
\text { corresponding to genes } R v 1918-R v 1963\end{array}$ & & $\begin{array}{l}\text { R. Brosch, unpublished data; } \\
\text { Glickman et al. (2001) }\end{array}$ \\
\hline M. tuberculosis $\mathrm{H} 37 \mathrm{Rv}$ & & Type strain of the $M$. tuberculosis complex & + & VLA Weybridge stock \\
\hline M. tuberculosis $\mathrm{Tb} 12$ & & Bangladeshi clinical isolate & + & Banu et al. (2004) \\
\hline M. tuberculosis Tb30 & & Bangladeshi clinical isolate & + & Banu et al. (2004) \\
\hline M. canettił & & Reference strain 140010059 & + & $\begin{array}{l}\text { Institut Pasteur stock; Gutierrez } \\
\text { et al. (2005) }\end{array}$ \\
\hline M. bovis type 9 & SB0140 & Sequenced strain (Garnier et al., 2003) & + & VLA Weybridge stock, AF2122/97 \\
\hline M. bovis type 17 & SB0263 & & - & $\begin{array}{l}\text { VLA Weybridge stock, 61/3139/ } \\
06\end{array}$ \\
\hline M. bovis type 17 & SB0263 & & - & $\begin{array}{l}\text { VLA Weybridge stock, 21/7917/ } \\
05\end{array}$ \\
\hline M. bovis type $17::$ IE471 & & $\begin{array}{l}\text { Type } 17 \text { strain transformed with cosmid } \\
\text { IE471 (described above) }\end{array}$ & + & This paper \\
\hline M. bovis type $17::$ pYUB4 12 & & $\begin{array}{l}\text { Type } 17 \text { strain transformed with empty } \\
\text { cosmid control }\end{array}$ & - & This paper \\
\hline M. bovis type 35 & SB0134 & $\begin{array}{l}\text { Field isolate with different spoligotype } \\
\text { from sequenced strain }\end{array}$ & + & $\begin{array}{l}\text { VLA Weybridge stock, 61/1307/ } \\
01\end{array}$ \\
\hline
\end{tabular}

${ }^{\star} \mathrm{SB}$, Spoligotype Bovis.

$\dagger+$, Region present; -, region not present.

¥The data obtained with $M$. tuberculosis Canetti ('M. canetti') are presented only in the supplementary tables. 
the empty pYUB412 vector or the pYUB412-based cosmid IE471, hygromycin was added to the medium up to $100 \mu \mathrm{g} \mathrm{ml}^{-1}$ as required. Cultures $(100 \mathrm{ml})$ in 21 capacity Greiner Bio-One bottles were grown at $37{ }^{\circ} \mathrm{C}$ and were aerated by rolling at 2 r.p.m. Growth rates, using pyruvate or Tween 80 , typically of $0.033 \mathrm{~h}^{-1}$ (range with pyruvate: $0.029 \mathrm{~h}^{-1}$ to $0.040 \mathrm{~h}^{-1}$, range with Tween $80: 0.030 \mathrm{~h}^{-1}$ to $0.035 \mathrm{~h}^{-1}$ ) were obtained with all the strains listed in Table 2 except $M$. bovis type 35 , which grew at $0.023 \mathrm{~h}^{-1}$ to $0.026 \mathrm{~h}^{-1}$ with either carbon source. The variation was between groups of batches; within each group the growth rates were very similar, except for that of $M$. bovis type 35 , which was notably slower. Thus the objective was largely achieved across a wide range of strains. Regardless of the medium that was used, $M$. bovis type 35 always grew more slowly than the other strains in Table 2 . Both growth rates $\left(<0.022 \mathrm{~h}^{-1}\right)$ and yields were much lower $\left[<10 \mathrm{mg}\right.$ dry weight $\left.(100 \mathrm{ml} \text { culture })^{-1}\right]$ for all strains when propanoate was the sole carbon source.

The decision not to use detergent in the medium so that culture filtrate lipids could be analysed meant that the cultures were of a granular appearance and could not be monitored by their $\mathrm{OD}_{600}$ values. However, samples taken at two or three intervals during growth, heat-killed, and dried revealed that their weights were similar to those in a duplicate culture including $0.025 \%(\mathrm{w} / \mathrm{v})$ of the detergent tyloxapol. Therefore, growth was monitored in the duplicate, parallel detergent-containing cultures. The tyloxapol did not support any growth. Yields of $36 \mathrm{mg}$ (dry wt) per $100 \mathrm{ml}$ culture could be obtained readily, except for M. bovis type 35, which gave $25 \mathrm{mg}$ (dry wt) per $100 \mathrm{ml}$. However, such cultures were entering lag phase and all metabolic labelling was done at mid-exponential phase, when the $\mathrm{OD}_{600}$ in parallel, detergent-containing cultures was 0.4 to 0.6 . These $\mathrm{OD}_{600}$ values equated to 12 to $18 \mathrm{mg}$ (dry wt) per $100 \mathrm{ml}$ for every strain in Table 2.

For construction of recombinant $M$. bovis type 17 knock-ins, the IE471 cosmid was selected from a M. tuberculosis Erdman library of pYUB412 integrating shuttle cosmids (Bange et al., 1999; Glickman et al., 2001) by hybridization with PCR-amplified probes from the $M b 1963 c-M b 1971$ region and sequencing of the insert termini following a previously published strategy (Brosch et al., 1998). Highly concentrated cosmid DNA from clone IE471 containing a $42 \mathrm{~kb}$ insert of $M$. tuberculosis Erdman DNA that corresponds to genes $R v 1918-R v 1963$ was then electroporated into M. bovis type 17 electrocompetent cells, where it stably inserted into the attB site situated in the glyV-tRNA gene. The same procedure was used for the pYUB412 vector control. Hygromycin-resistant transformants appearing after 4 weeks on Middlebrook 7H11 medium (Difco) supplemented with oleic acid-albumin-dextrose-catalase (OADC, Difco) and $100 \mu \mathrm{g}$ hygromycin $\mathrm{ml}^{-1}$ were analysed for the presence of genes orthologous to $M b 1963 c-M b 1971$ by specific amplification and sequencing. The resulting $M$. bovis type $17:$ IE471 and the $M$. bovis type $17:$ pYUB412 vector control strains were used for further biochemical analyses.

Labelling with acetate or propanoate. Cultures growing at the same rate (as near as possible), during exponential phase, were labelled with $\left[1-{ }^{14} \mathrm{C}\right]$ acetate or $\left[1-{ }^{14} \mathrm{C}\right]$ propanoate, to minimize any differences in metabolism due to growth rate or phase. The density of bacteria, 12 to $18 \mathrm{mg}$ (dry wt) per $100 \mathrm{ml}$, was chosen to maximize the yield of exponential-phase bacteria. All labelling was done for $2 \mathrm{~h}$ with $50 \mu \mathrm{Ci}(1850 \mathrm{kBq})$ sodium salt, 50 to $60 \mathrm{Ci} \mathrm{mol}^{-1}$, per $100 \mathrm{ml}$ medium, adding the labelled compound directly to cultures and incubating in growth medium and conditions. Radioisotopically labelled substrates were from American Radiolabelled Chemicals (ARC 101A for acetate; ARC 203A for propanoate). The main conclusions of this work were drawn from labelling followed by analysis - 'pulse-only' experiments. As part of our global analysis of lipid labelling, some pulse-chase experiments were done. For these, a
$10 \mathrm{ml}$ sample was analysed at the end of the $2 \mathrm{~h}$ pulse phase and the remainder of the suspension was washed again in medium and resuspended in $90 \mathrm{ml}$ medium free of labelled compounds for a chase phase. After $4 \mathrm{~h}$ and $20 \mathrm{~h}$ chase phase, $45 \mathrm{ml}$ samples were taken.

Lipid analysis. After $2 \mathrm{~h}$, the bacteria were harvested by centrifugation and washed once in MilliQ water. When pulse-chase experiments were done, additionally, the samples after the $4 \mathrm{~h}$ and $20 \mathrm{~h}$ chase were also harvested by centrifugation and washed once in MilliQ water. In most of the experiments, superficial lipids were extracted with $0.05 \%$ decylamine in hexane (Converse et al., 2003), the unbound lipids were extracted with successive mixtures of hexane and chloroform/methanol/water mixtures to give 'non-polar' and polar lipid fractions (Besra, 1998; Dobson et al., 1985), and the wallbound mycolic acids were released from the remaining residue with $15 \%$ (w/v) tetrabutylammonium hydroxide (TBAH) at $100{ }^{\circ} \mathrm{C}$ for $18 \mathrm{~h}$. The TBAH salts thus produced were then methylated and analysed by TLC (Fig. 1c) (Wheeler, 2008).

Most of the label in the mycolates was in the wall-bound fraction. However, during the global analysis of metabolic labelling of lipids that was conducted in this study, minor amounts of mycolate were detected in the unbound lipids (see Supplementary Tables S1 and S2, available with the online version of this paper). Unbound lipids were analysed by successive 2D TLC (Besra, 1998; Dobson et al., 1985). The unbound mycolate-containing lipids are shown in Fig. 1(a,b) with the solvent systems used to develop them.

The amount of radioactivity in wall-bound mycolates was determined by sampling $10 \%$ of the volume of the methyl esters dissolved in hexane and scintillation counting in Scintran NA (VWR). Samples containing 30000 to 100000 d.p.m. were applied to the concentrating zone of Silica Gel 60 TLC plates (Merck 13748) and the plates developed three times in hexane/ethyl acetate $(95 / 5, \mathrm{v} / \mathrm{v})$. The amount of radioactivity in each band on TLC plates (Fig. 1c) was quantified using a phosphorimager or by scraping off individual radioactive spots. These two methods gave values that agreed. The total amount in the wall-bound mycolates was then calculated by working out the proportion of radioactivity in the bands corresponding to mycolates (Fig. 1c) and multiplying by the value for all of the material dissolved in hexane.

A step-by-step protocol for the labelling and global lipid analysis performed in this work is available (Wheeler, 2008; Wheeler \& Anderson, 1996).

Lipid and pyruvate analysis after quenching metabolism: experiments 1150 and 1160. In order to analyse the pyruvate formed from labelled substrates, metabolism had to be rapidly quenched. This was achieved by pipetting $20 \mathrm{ml}$ samples of cultures, after $2 \mathrm{~h}$ labelling, into $20 \mathrm{ml}$ methanol at $-80{ }^{\circ} \mathrm{C}$. The mixture was kept on dry ice in centrifuge buckets pre-cooled to $-80{ }^{\circ} \mathrm{C}$ and transferred quickly into a pre-cooled Sigma $3 \mathrm{~K} 10$ centrifuge set at $-20{ }^{\circ} \mathrm{C}$ and centrifuged for $8 \mathrm{~min}$ at $4800 \mathrm{~g}$. The pellet was recovered by removing all the supernatant while the buckets were kept on dry ice and stored at $-80{ }^{\circ} \mathrm{C}$. Labelled keto acids were extracted from the pellet by adding $1 \mathrm{ml}$ methanol at $-80{ }^{\circ} \mathrm{C}$, freezing by plunging into liquid nitrogen and thawing on dry ice three times, collecting the pellet and supernatant by centrifuging in the pre-cooled centrifuge, repeating the extraction using $0.5 \mathrm{ml}$, and pelleting and combining the two supernatants. The supernatants were freeze-dried at $-80{ }^{\circ} \mathrm{C}$, then $330 \mu \mathrm{l} 25 \mathrm{mM}$ 1,2-diamino-4,5-dimethoxybenzene (DDB) in $1 \mathrm{M} \mathrm{HCl}$ was added directly to the dried material. DDB derivatives of the keto acids were recovered and their radioactivity analysed as described previously (Wheeler et al., 2005). The pellets were washed twice at $25{ }^{\circ} \mathrm{C}$ (room temperature: not critical) with $2 \mathrm{ml}$ chloroform/methanol $(2: 1, \mathrm{v} / \mathrm{v})$; their wall-bound mycolates were prepared as methyl esters (MAMEs), and their radioactivity was quantified and analysed as described above. 
(a)

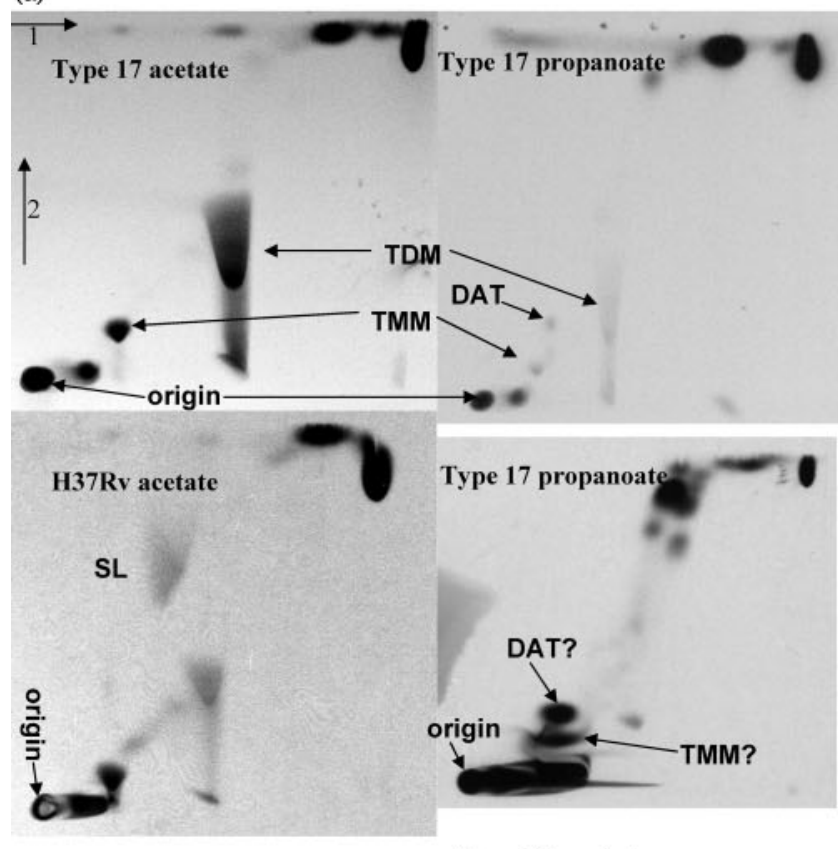

(b)

Type 17 acetate

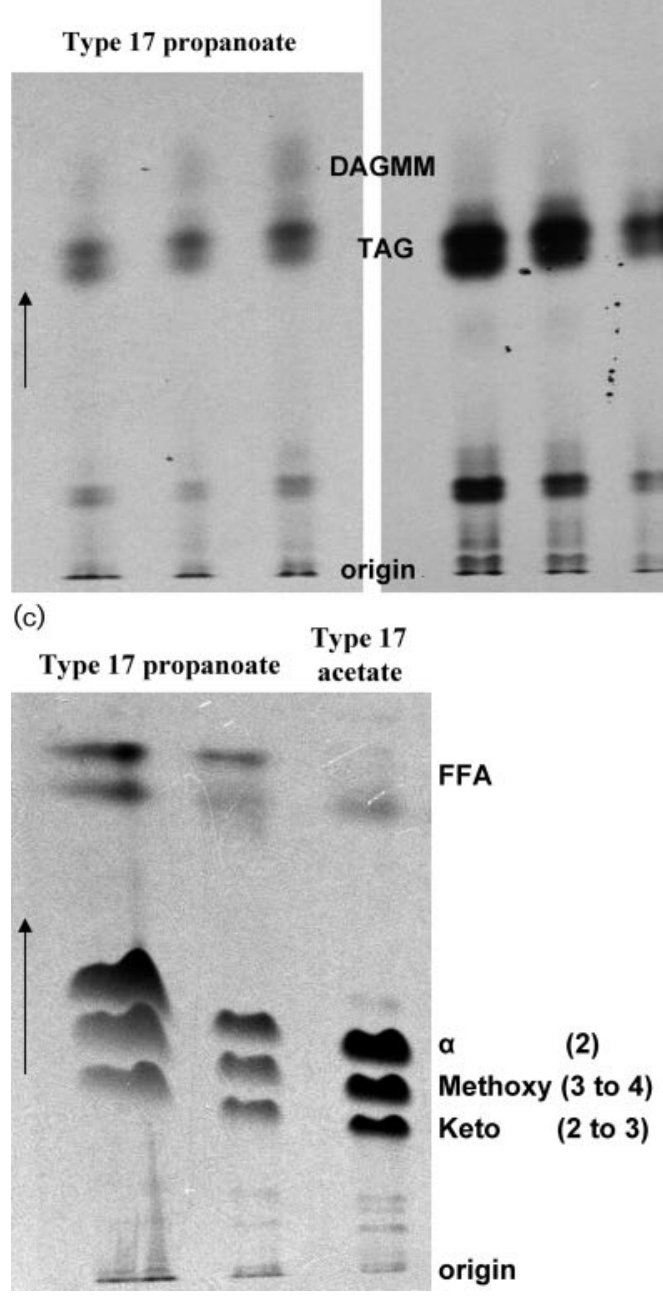

Fig. 1. TLCs of all mycolate-containing lipids. Directions of solvent development are shown with arrows that apply to all TLCs in that panel. (a) 2D TLC by system D (Dobson et al., 1985), developed in direction 1 with chloroform/methanol/water ( $250: 35: 2$, by vol.) then in direction 2 with chloroform/acetone/ methanol/water (100:120:5:6, by vol.). Lipids were extracted from bacteria immediately after the pulse phase. The unbound 'non-polar' lipid fraction was applied to the two top plates and the bottom left plate. The bottom right plate shows trehalose monomycolate (TMM) and diacyltrehalose (DAT) from unbound polar lipids in a very congested area that was difficult to resolve. All other lipids were accurately quantified. (b) DAGMMs labelled with propanoate and acetate. The percentage of label in DAGMMs was 2.5 to 4 times higher in the propanoate-labelled DAGMMs. Spots labelled TAG are triacylglycerols that do not have a mycolyl group. Solvent system: toluene/acetone (124:1, v/v). Samples are, left to right: immediately after the pulse phase; $4 \mathrm{~h}$ chase; $20 \mathrm{~h}$ chase. Unbound lipids are shown in (a) and (b). (c) Labelling of the three classes of mycolates. Material released from cell wall residue washed free of unbound lipids by alkaline hydrolysis, then methylated, was applied to TLC plates and developed with hexane/ethyl acetate $(95: 5, \mathrm{v} / \mathrm{v})$ run three times with fresh solvents being used for each run and drying of plates between runs. The acetate-labelled material (right-hand lane: before the chase phase is shown) is richer in mycolates than the propanoatelabelled material (two lanes shown: immediately after the pulse phase, and $20 \mathrm{~h}$ chase). The number of methylation reactions required during their biosynthesis is given in parentheses: for ketoand methoxymycolates, the lower values are for cis mycolates, the higher for trans mycolates, which are approximately equally represented but not separated by the TLC system. FFA, free fatty acids.

Statistics and expression of data. Values of d.p.m. per mg dry weight of bacteria were calculated from the d.p.m. and dry weight data for each sample; numbers of determinations are shown in the tables. Examples of d.p.m. $\mathrm{mg}^{-1}$ values are given in Fig. 2(b); all values are in the supplementary tables. The ratio of d.p.m. per mg dry weight of bacteria in mycolates labelled with acetate: propanoate were calculated for each pair of samples done in parallel, i.e. in the same experiment, and their mean values \pm SEM are presented. Fisher's exact $t$-test was conducted on the ratio values to test for statistical differences of the ratios. The percentage of label in each lipid was calculated (i) relative to the d.p.m. $\mathrm{mg}^{-1}$ values after the pulse phase, with no chase, and (ii) relative to the label in each sample. Linked spreadsheets with each of these sets of percentage values are given in Supplementary Tables S1 and S2. Percentage values thus calculated were used in the Results section, with SEM values calculated on the percentage values.

\section{RESULTS}

\section{Global metabolic labelling of lipids}

Mycobacterial lipids were labelled and the distribution of label followed in pulse-chase experiments for all lipids. The full data for labelling of unbound, non-polar lipids are included in Supplementary Table S1. Those data failed to show any differences in the unbound lipids that could be 
(a)
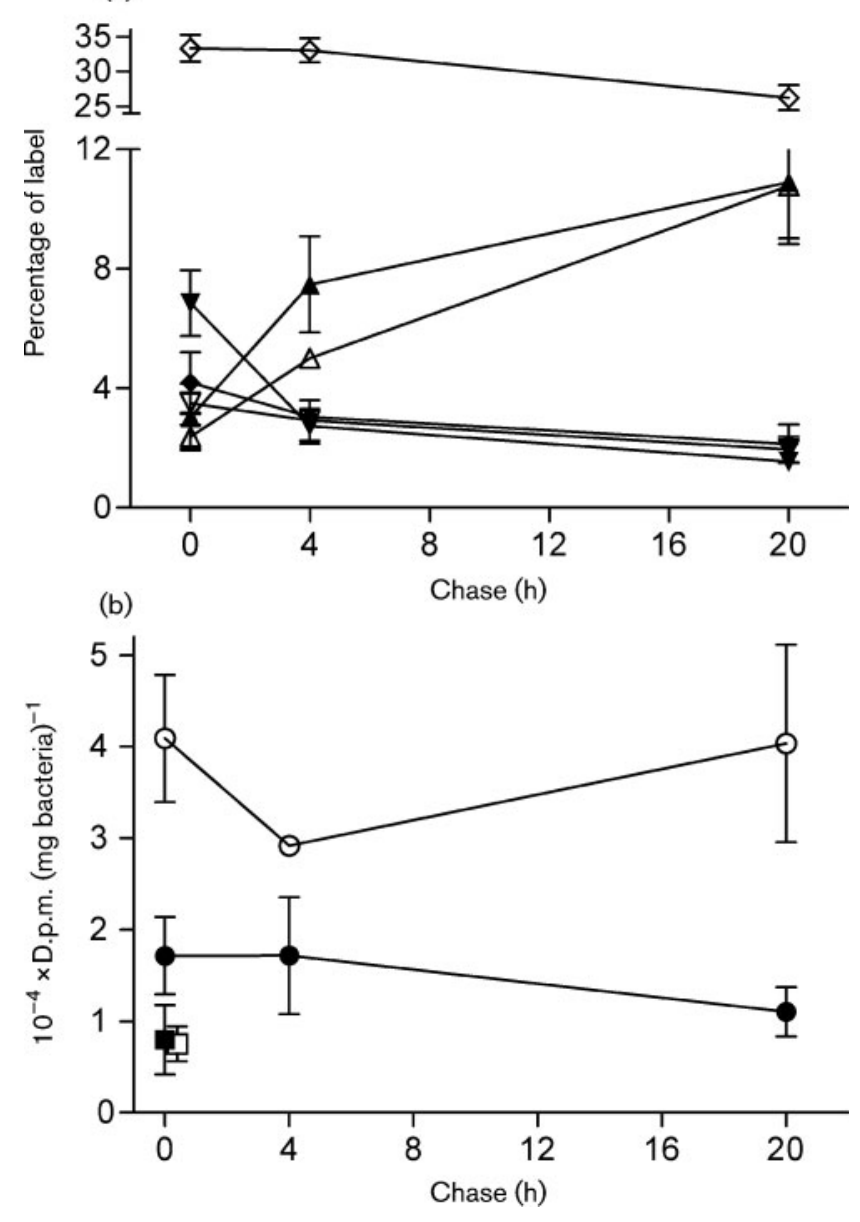

Fig. 2. Labelling with acetate and chase of all mycolates. Filled symbols are used for $M$. tuberculosis; open symbols are used for $M$. bovis. M. tuberculosis H37Rv and its pYUB412 construct were included in calculating mean values for acetate incorporation at each time point as there were no consistent differences in this activity when the cosmid was present. In (a), unbound lipids are shown: TMM ( $\boldsymbol{\nabla}$, $\nabla), \operatorname{TDM}(\boldsymbol{\Delta}, \triangle)$, TAG $(\diamond, \diamond)$. TAG is included in this graph as it represented the lipid from which most label was chased out over the $20 \mathrm{~h}$ chase. All values are percentage of label in each sample \pm SEM; e.g. after $20 \mathrm{~h}$ chase the value is relative to the label in the bacteria after $20 \mathrm{~h}$. The means for $M$. bovis are calculated from values obtained with all the $M$. bovis spoligotypes used in this study as their incorporation of acetate into each of TMM, TDM and TAG was similar. All data from pulse-chase experiments are shown. In (b) wall-bound mycolates are shown: bacteria grown with pyruvate $(\bullet, \bigcirc)$; bacteria grown with Tween $80(\square, \square)$. The values from $M$. bovis type 17 grown on Tween 80 as the sole carbon source are nudged $0.4 \mathrm{~h}$ for clarity, but represent labelling from cultures pulsed with acetate, and not chased. The means for $M$. bovis were calculated from values obtained with $M$. bovis type 17 and its construct, $M$. bovis type $17:$ : pYUB412, containing an empty cosmid vector, as there were no consistent differences in this activity when the cosmid was present. All values are d.p.m. per mg (dry wt) bacteria \pm SEM. The different spoligotypes of $M$. bovis used in this work varied in their incorporation of acetate into wall-bound mycolates, so the data from one selected strain on which pulse-chase experiments was conducted is shown here; all other data are in Supplementary Table S2. associated with RDbovis(d)_0173. However, there were differences in the extent to which mycolic acids were labelled with propanoate and acetate; the data are presented in Supplementary Table S2.

\section{Distribution of the labelled mycolates}

Most of the metabolic labelling of mycolates by acetate was into wall-bound mycolates (mean of $79 \% \pm 1.3 \%$, SEM in all 38 determinations across all strains and time points) with nearly all the remaining $21 \%$ (range: $4 \%$ to $37 \%$ ) going into trehalose mycolates. Usually, only a trace was detected in triacylglycerols (TAGs) as di-non-hydroxyacyl, monomycolyl glycerol (DAGMM: mean of $1.85 \% \pm 0.44 \%$, SEM in all 14 determinations across all strains and time points). Apparently a greater proportion of the label from propanoate is incorporated into the trehalose mycolates, but labelling in the region of the TLC plates (Fig. 1a) around trehalose monomycolate (TMM) is very congested in the propanoatelabelled material so radioactivity values assigned to TMM are almost certain to be overestimates. Therefore, the labelling of wall-bound mycolates was used to assess the differential labelling with acetate and propanoate, since this is very clean material, derived from a cell residue washed free of all non-covalently bound lipids.

Surprisingly, given that propanoate labelled mycolates poorly, the proportion of labelling in TAGs in the mycolate-containing TAGs (DAGMMs) was usually higher with propanoate than with acetate (Fig. 1b). The values for the proportion of propanoate-labelled mycolate in DAGMMs, shown in Supplementary Table S2, must partly reflect there being so little label in the wall-bound mycolates, as it is high after labelling with propanoate of M. bovis type 17. Prenylphosphomannose-mycolate (Besra et al., 1994) should appear between TMM and TDM in the first direction of 2D TLC, but no unassigned labelled material was observed here during this study (Fig. 1a).

In pulse-chase experiments, label was chased out of TMMs and into TDMs (Fig. 2a). Label was also especially rapidly chased out of TAGs (Fig. 2a), though very little of this label is in mycolyl groups (Fig. 1b). There was a fall in specific activity (d.p.m. per mg dry weight bacteria) in most lipids (data in Supplementary Table S1) but label was chased into wall-bound mycolates at a rate consistent with growth. This is evident from the conservation of the specific activity in wall-bound mycolates in the chase samples (Fig. 2b) while the mass of bacteria increased over the $20 \mathrm{~h}$ period. Mean values of incorporation of acetate into mycolic acids are shown in Fig. 2(b) for every experiment done with $M$. tuberculosis $\mathrm{H} 37 \mathrm{Rv}$ and M. bovis type 17 (raw data in full for every strain under MAMEs in Supplementary Table S2).

\section{Labelling of mycolic acids with acetate and propanoate}

Acetate labelled mycolic acids much more efficiently than propanoate in all mycobacteria used in this study. 
However, relative to the labelling with acetate, labelling with propanoate was significantly lower in M. bovis type 17 . The relative efficiency of labelling is expressed as the ratio of labelling with acetate:propanoate (Table 3) so the relative efficiency of labelling with propanoate is the reciprocal of the values in Table 3. For the reasons stated above, wall-bound mycolates were used to calculate this ratio. In the wall-bound mycolic acids, the proportion of label in the three classes of mycolic acid was the same regardless of either the strain or whether the labelling was with acetate or propanoate (Fig. 1c). The values were 43 $46 \%$ of the label in $\alpha$-mycolates, $36-37 \%$ in methoxymycolates and $17-20 \%$ in ketomycolates. Even when the caveat about assigning d.p.m. $\mathrm{mg}^{-1}$ values to propanoatelabelled TMM was ignored and the ratio of propanoate: $\mathrm{a}-$ cetate labelling was calculated for all mycolates, i.e including the trehalose mycolates and DAGMMs, the ratio was higher in $M$. bovis type 17 strains, showing their less efficient labelling of total mycolates with propanoate (these ratio values are in Supplementary Table S2).

Especially compelling was the difference in the labelling of wall-bound mycolates between all type 17 field strains and the other strains of $M$. bovis when the data for type 9 and type 35 strains were combined (Table 3 ). These three spoligotypes are genetically extremely similar apart from type 17 having lost the RDbovis(d)_0173 region including three genes for lipid catabolism (Table 1). Taking the data from all experiments, the acetate:propanoate ratio was significantly lower for all other $M$. bovis strains $(P=0.016$ to 0.022$)$ and for every experiment done with $M$. tuberculosis H37Rv ( $P=0.007$ to 0.022 ). Even in the case of type 17 strain $21 / 7917 / 05$, that had a ratio of labelling with acetate:propanoate apparently intermediate between type 9 and the other type 17 strains, the difference was close to significant from type $9(P=0.062)$ but not $(P=0.144)$ from the type 17 strain 61/1121/01 [data and statistical analysis from experiment 1160 (Supplementary Table S2) when different field strains of spoligotype 17 were compared with type 9].

To determine whether this effect was related to the function of genes $M b 1963 c-M b 1971$, missing from type 17 M. bovis strains due to the RDbovis(d)_0173 deletion, recombinant $M$. bovis type 17 variants were constructed that carried an integrating shuttle cosmid (IE471) containing the M. tuberculosis orthologues of Mb1963c-Mb1971 and large portions of their flanking regions. The IE471clone was selected from a M. tuberculosis Erdman pYUB412 cosmid library (Bange et al., 1999; Glickman et al., 2001) by hybridization and sequencing of the insert termini following a previously published strategy (Brosch et al., 1998).

\section{Restoration of labelling of mycolic acids with propanoate by Mb1963c-Mb1971 orthologues}

The M. bovis type $17:$ IE471 construct that includes the Mb1963c-Mb1971 orthologues (Table 2) restored the acetate:propanoate labelling ratio to that of other $M$. bovis strains. In the experiment in which this construct was tested along with all the other strains shown in Table 3 (experiment 1150: the raw data are shown in Supplementary Table S2), the acetate: propanoate labelling ratio was lower than for the other $M$. bovis strains and comparable with the human strains, Tb12 and H37Rv. The ratio in $M$. bovis type $17::$ IE471 (Table 3) was significantly lower than in the 'empty vector' control, M. bovis type

Table 3. Acetate : propanoate labelling ratios of wall-bound mycolic acids

\begin{tabular}{|lcc|}
\hline Strain & All experiments & Quenched experiments only $\dagger$ \\
\hline M. tuberculosis $\mathrm{H} 37 \mathrm{Rv}$ & $6.50 \pm 0.66(14)$ & $7.70 \pm 0.58(3)$ \\
M. tuberculosis $\mathrm{Tb} 12$ & $5.37 \pm 0.98(5)$ & $7.84 \pm 0.44(2)$ \\
M. tuberculosis $\mathrm{Tb} 30$ & $2.88 \pm 0.14(3)$ & Not done $\ddagger$ \\
M. bovis type $9 \mathrm{AF} 2122 / 97$ & $7.61 \pm 0.76(8)$ & $7.95 \pm 0.93(6)$ \\
M. bovis type $1761 / 1121 / 01$ & $20.34 \pm 3.77(8)$ & $19.68 \pm 1.90(3)$ \\
M. bovis type $1761 / 3139 / 06$ & $22.56 \pm 1.54(3)$ & $22.56 \pm 1.54(3)$ \\
M. bovis type 17 21/7917/05 & $11.39 \pm 1.44(3)$ & $11.39 \pm 1.44(3)$ \\
M. bovis type $17:: \mathrm{IE} 471$ & $9.61 \pm 1.36(5)$ & $7.43 \pm 0.40(3)$ \\
$M$. bovis type $17:: \mathrm{pYUB} 412$ & $20.48 \pm 2.99(5)$ & Not done \\
$M$. bovis type 35 61/1307/01 & $7.30 \pm 1.84(5)$ & \\
\hline
\end{tabular}

*All time points were included in calculating these ratios as d.p.m. (mg bacteria) ${ }^{-1}$, as ratio values did not show any pattern of increase or decrease with time. The mean of the values \pm SEM (number of ratios calculated from data) is shown.

$\dagger$ Experiments in which labelling of intracellular pyruvate and wall-bound mycolates were determined in the same samples of bacteria; only a pulse phase was done in these experiments, followed by rapid quenching of metabolism to preserve intracellular metabolites. These are referred to as experiments 1150 and 1160 in Supplementary Table S2.

‡Ratio not significantly different between Tb12 and Tb30 in experiments where both strains were labelled (see Supplementary Table S2). 
$17::$ pYUB412 $(P=0.041)$. The general pattern of restoration of more efficient propanoate labelling of mycolates was evident from the ratios obtained in every experiment in which the two constructs were tested ('all experiments' in Table 3), and also in experiments in which intracellular metabolites as well as wall-bound mycolates could be determined in the same sample ('Quenched experiments only' in Table 3). As propanoate is expected to be converted to either acetyl-CoA, which would label mycolates, or to pyruvate, as suggested by examination of likely metabolic pathways, the labelling of pyruvate was also determined in the quenched experiments.

\section{Propanoate is incorporated more efficiently into pyruvate in $M$. bovis type 17}

The metabolic labelling of pyruvate with propanoate was around four times higher in M. bovis type 17 than in $M$. bovis type $17:$ :IE471 (Table 4). Labelling of pyruvate was very similar $(P=0.38)$ in the type 17 field strain and type $17::$ pYUB412 (with the empty vector introduced), giving the option of combining these data as the 'Type 17 aggregate' for significance testing (Table 4). The M. bovis type 17 aggregate incorporated label from propanoate into pyruvate significantly more efficiently $(P$ values are in Table 4) than M. bovis type $17:$ :IE471, the other M. bovis strains tested, and M. tuberculosis. When metabolic labelling of pyruvate with propanoate was compared with two other field strains of type 17, the labelling was indistinguishable between the type 17 strains but significantly different from the labelling in type 9 (Table 4). These data may explain the inefficient incorporation of label from propanoate into mycolate by $M$. bovis type 17 as a result of its diversion to pyruvate (Fig. 3), an effect that is reversed when the genes deleted in RDbovis(d)_0173 are restored. Note that d.p.m. $\mathrm{mg}^{-1}$ values are given in this section because label from acetate was not incorporated into pyruvate and therefore no ratio could be calculated.

Given that label from propanoate was incorporated into pyruvate as an alternative to acetyl-CoA, it would have appeared to be preferable to grow the bacteria with an alternative carbon source to pyruvate. However, the only alternative carbon sources that all the strains in Table 2 can be grown on is a lipid, or propanoate itself. All the strains grew on Tween 80 , a source of oleate, at similar rates to their growth on pyruvate. However, when they were pulsed with either labelled acetate or propanoate, incorporation of label into lipids was three to six times lower than when they were grown, in parallel, on pyruvate. As an example, data of all incorporation values obtained in this work are shown in Fig. 2(b) for all $M$. tuberculosis constructs and all the M. bovis type 17 constructs. Fatty acids such as oleate are good feedback inhibitors of de novo fatty acid biosynthesis (Bloch, 1977) and this effect was previously demonstrated when it was shown that lipids in culture media depressed acetate incorporation into lipids by up to 10 -fold (Wheeler \& Ratledge, 1988). Propanoate as a sole carbon source (in Sauton medium) supported the growth of all the strains in Table 2, although on subculturing from Sauton medium with pyruvate as sole carbon source, or Middlebrook 7H9 with ADC, Tween 80 and pyruvate, lag phases of 3 to 6 weeks occurred. On subculturing from Sauton with propanoate, lag phases were always less than 2 weeks but the maximum growth yield was $10 \mathrm{mg}$ (dry wt) per $100 \mathrm{ml}$ culture medium, and growth rates varied between the strains (fastest $\sim 0.022 \mathrm{~h}^{-1}$ ). Together with the problem that labelled propanoate would have been diluted out with unlabelled propanoate, at $2 \mathrm{~g} \mathrm{l}^{-1}$, to support growth, propanoate too proved to be unsuitable as a carbon source in these labelling experiments.

Table 4. Incorporation of label from propanoate into pyruvate

All values (means \pm SEM) were from three determinations, except for M. bovis type 9 AF2122/97 (six) and the M. bovis type 17 aggregate results, which were calculated from data from the type 17 field strains and M. bovis type $17::$ pYUB412 combined. These sets of data for the different type 17 strains were not significantly different (for pairwise comparisons: $P>0.38$ ).

\begin{tabular}{|c|c|c|}
\hline Strain & D.p.m. $(\text { mg bacteria })^{-1}$ & $P$ vs $M$. bovis type 17 aggregate \\
\hline M. tuberculosis $\mathrm{H} 37 \mathrm{Rv}$ & $1983 \pm 258$ & 0.021 \\
\hline M. tuberculosis $\mathrm{Tb} 12$ & $1216 \pm 313$ & 0.010 \\
\hline M. bovis type 9 AF2122/97 & $2368 \pm 248$ & 0.010 \\
\hline M. bovis type $1761 / 1121 / 01$ & $3522 \pm 471$ & \\
\hline M. bovis type $1761 / 3139 / 06$ & $3454 \pm 548$ & \\
\hline M. bovis type 17 21/7917/05 & $3575 \pm 45$ & \\
\hline M. bovis type $17::$ IE471 & $835 \pm 146$ & \\
\hline M. bovis type $17:$ :pYUB412 & $2887 \pm 170^{*}$ & \\
\hline M. bovis type 17 aggregate & $3516 \pm 237$ & \\
\hline
\end{tabular}

${ }^{\star}$ Significantly different from value for $M$. bovis type $17::$ IE471 $(P=0.002)$. 


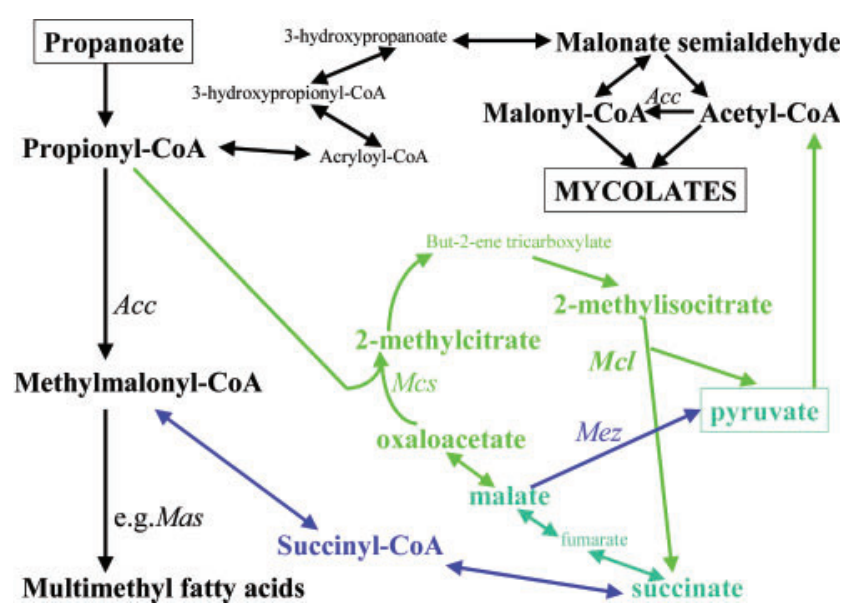

Fig. 3. Metabolic fate of propanoate. Pathways leading to acetyl$\mathrm{CoA}$ and methylmalonyl-CoA, needed for lipid biosynthesis, are in black. The methylcitrate cycle, for generating pyruvate, is shown in green. An alternative possible route for generating pyruvate is shown in blue, with shared reactions in turquoise: acetyl-CoA could be generated through either of these two routes, too, by decarboxylation of pyruvate. Acc, acyl-CoA carboxylase; Mas, mycocerosic acid synthase; Mcl, methylisocitrate lyase; Mcs, methylcitrate synthase; Mez, malic enzyme.

\section{DISCUSSION}

Bovine tuberculosis is one of the most difficult animal health problems that the British farming industry faces. The number of cattle infected with bovine tuberculosis has been increasing year on year by $18 \%$, which leads to serious losses for affected farms due to the slaughter of infected animals and the imposition of cattle movement restrictions. One possibility is that new forms of M. bovis have evolved in GB that are able to circumvent the current control measures. Evidence for this latter scenario is provided by the presence of a range of different types of $M$. bovis circulating in GB that seem to be successful in spreading around the country from their original place of isolation. The hallmark of this clonal expansion is provided by the $M$. bovis type 17 clone, which accounts for more than $30 \%$ of all clinical isolates of M. bovis. By determining the molecular basis of the success of this strain we hope to understand the selective forces that drive the emergence of new strains. As mycobacterial lipids have been shown to be key immunomodulators (Reed et al., 2004; Sinsimer et al., 2008), we were intrigued by the clonal deletion of genes involved in lipid metabolism from type 17 strains, and sought to determine the effect of this deletion on surface lipid profiles of type 17 .

Mycobacterial lipids can be readily labelled during growth of the bacteria with radioactive acetate or propanoate. Usually one carbon source gives optimal labelling. When polyketide synthases are involved in the biosynthesis of a lipid, with the incorporation of methyl branches in its fatty acyl moieties, propanoate is the best labelled carbon source
(Minnikin et al., 2002; see also Supplementary Table S1). However, when methyl branches are not present in the fatty acids, or if present are not derived from methylmalonyl-CoA, label from propanoate cannot be directly incorporated into the lipid, as in the case of mycolic acids. Mycolic acids are 1-alkyl 2-hydroxy fatty acids. Their biosynthetic pathways have been worked out: their acyl chains are built up solely from acetyl-CoA (Marrakchi et al., 2008) and any methyl groups are derived from $S$ adenosylmethionine using Mmas, Cmas and related methyltransferases (Marrakchi et al., 2008). Nevertheless, incorporation of label from propanoate into mycolates was observed in this study. Some strains were quite efficient at labelling with propanoate but $M$. bovis type 17 was remarkably inefficient and we wondered if this might be related to its loss of some fadE and echA genes (Table 1). Therefore, we analysed the relative incorporation of label from acetate and propanoate into mycolates further. Given that metabolic fingerprints of $M$. bovis cluster with molecular type (Winder et al., 2006) but the actual metabolites were not identified, we conducted some identification of metabolites labelled in this study. We targeted keto acids as they include pyruvate - a key metabolite - and tricarboxylic acid cycle intermediates, and are likely to be derived from propanoate (Fig. 3).

Our data can be rationalized from consideration of metabolic pathways and our knowledge, outlined above, of mycolate biosynthesis. Propanoate must be metabolized first to acetyl-CoA to initiate mycolate biosynthesis and for malonyl-CoA to be incorporated into the growing acyl chains of mycolates (Fig. 3). It is just possible that the carbon from propanoate is incorporated only into the methyl groups of mycolic acids, or via some other route than via acetyl-CoA, although any pathways or metabolic nodes would be highly tenuous. However, experimental support for propanoate being converted to acetyl-CoA was obtained in this work from the observation that it labelled the three classes of mycolates (with different degrees of methylation required to form each of them) in the same proportion as when acetate was used as the label (Fig. 1c). The ability to convert propanoate to acetyl-CoA and malonyl-CoA is indeed a prerequisite for growth on propanoate as a single carbon source, a growth property demonstrated both here and by McKinney's group (Munoz-Elias et al., 2006; Savvi et al., 2008). It is probably also needed to remove excess propanoate generated by catabolism of odd-chain fatty acids from metabolic pools, as this acid is toxic (Savvi et al., 2008). The likely pathways are shown in Fig. 3; the route to pyruvate is the methylcitrate cycle as outlined previously (MunozElias et al., 2006) and acetyl-CoA can be generated from the pyruvate. Alternatively, to generate acetyl-CoA or malonylCoA, a new pathway via malonate semialdehyde is proposed (Fig. 3). The overall reactions for both pathways are:

Propanoate $+\mathrm{ATP}+\mathrm{CoA}+2 \mathrm{NAD}(\mathrm{P})^{+}+\mathrm{FAD}=$ Malonyl $\mathrm{CoA}+\mathrm{AMP}+2 \mathrm{P}_{\mathrm{i}}+2 \mathrm{NAD}(\mathrm{P}) \mathrm{H}+\mathrm{FADH}_{2}+2 \mathrm{H}^{+}$

Acetyl-CoA is generated by the same pathway initially with 
the same reduction and phosphorylation cost of coenzymes, with the additional generation of $\mathrm{CO}_{2}$. Both pathways are feasible as they are less costly in energy than the generation of methylmalonyl-CoA, which has been demonstrated directly (Rainwater \& Kolattukudy, 1982):

Propanoate $+2 \mathrm{ATP}+\mathrm{CoA}+\mathrm{CO}_{2}=$ Methylmalonyl -

$\mathrm{CoA}+\mathrm{AMP}+\mathrm{ADP}+3 \mathrm{P}_{\mathrm{i}}$

The closeness of the relationship of the M. bovis strains suggested that it might be the major genetic difference between them, the loss of genes Mb1963c-Mb1971 (Table 1 ), that gives rise to this phenotype, especially as three of the genes were predicted to be involved in lipid catabolism (Table 1). This was confirmed by adding back this genetic region to $M$. bovis type 17 and restoring the incorporation of label from propanoate to levels comparable with all the other strains surveyed that had the $M b 1963 c-M b 1971$ genes or their orthologues. If, in $M$. bovis type 17 , very little propanoate was channelled to acetyl-CoA, a feasible alternative metabolic fate would be to pyruvate (Fig. 3). As well as being a feasible pathway that is not contrived, this was an attractive scenario because all $M$. bovis field strains lack alanine dehydogenase (Chen et al., 2003) and pyruvate kinase (Keating et al., 2005), with their respective ald and $p y k A$ genes being inactive (Garnier et al., 2003). In these strains, pyruvate would have to be acquired from an exogenous source or by anaplerotic reactions using the acetyl-CoA generated from $\beta$-oxidation of fatty acids via the glyoxylate cycle and malic enzyme (Mez). Therefore a genetic change that led to further compensation of the lesions in pyruvate production might be selectable in new conditions for $M$. bovis. When it was tested whether the loss of the Mb1963c-Mb1971 genes or their orthologues led to the production of pyruvate, $M$. bovis type 17 and type $17::$ pYUB412 (the empty vector control) produced significantly more labelled pyruvate from labelled propanoate than the isogenic type $17:$ IE471 with the genes added back in, or any of the non-isogenic strains with the Mb1963c-Mb1971 genes or their orthologous genes. The pathways in Fig. 3 show the methylcitrate cycle for generating pyruvate, a cycle that uses bifunctional isocitrate lyases for the $\mathrm{Mcl}$ (methylisocitrate lyase) step and that is needed for M. tuberculosis to grow on propanoate (Gould et al., 2006; Munoz-Elias et al., 2006). A feasible alternative that could possibly be used if methylmalonyl-CoA (Savvi et al., 2008) is produced in excess is shown in blue in Fig. 3. This would also allow malate, a product of the glyoxylate cycle, to be used for pyruvate production. Both have the same overall reaction:

Propanoate $+\mathrm{XTP}+\mathrm{FAD}+\mathrm{NAD}^{+}=$Pyruvate $+\mathrm{XMP}+$ $2 \mathrm{P}_{\mathrm{i}}+\mathrm{FADH}_{2}+\mathrm{NADH}+\mathrm{H}^{+}$

Although the complementation experiment confirmed the role of the genes $M b 1963 c-M b 1971$ in propanoate metabolism, this work does not define the mechanism of their action. It is unlikely that any of these genes encode enzymes for the steps in the pathways in Fig. 3, although the genes for the two enzymes for generating, then oxidizing, malonate semialdehyde have not been annotated in the M. tuberculosis complex genomes and Mb1964c is a probable dehydrogenase (Table 2). However, the FadE and EchA enzymes might generate fatty acyl-CoAs or their derivatives that could affect the pathways as these are powerful regulatory compounds at both transcriptional and allosteric levels (Bloch, 1977; Flick \& Bloch, 1975; Iram \& Cronan, 2005; van Aalten et al., 2001). Further, Mb1966c is predicted to be a transcriptional regulatory protein, and may play a role in regulating the pathways in Fig. 3

Previous studies have linked subtle changes in mycolic acid structure to virulence. However, in this study differential labelling of mycolic acids with propanoate across strains of $M$. bovis and the $M$. tuberculosis complex revealed a difference in the central pathways of carbon metabolism that was related to the loss of RDbovis(d)_0173. This region, lost in $M$. bovis type 17, includes lipid catabolism genes and a possible transcriptional regulator. We suggest that the loss of the RDbovis(d)_0173 leads to very little propanoate being channelled into acetyl-CoA (for mycolate biosynthesis) but more being channelled into pyruvate, using the central pathways illustrated in Fig. 3. Given the need for M. bovis to compensate for natural mutations in ald and $p y k A$ to replenish its intracellular pool of pyruvate, this additional compensatory mechanism may confer an advantage to $M$. bovis type 17 and may contribute to its extraordinary expansion in a microepidemic of bovine tuberculosis.

\section{ACKNOWLEDGEMENTS}

This work was funded by the Department for Environment, Food \& Rural Affairs (UK) (Defra). We thank Jim Dale for curation and making available the type 17 field strains, W. R. Jacobs, Jr, for an aliquot of the pYUB412 library and Lynn Dover for invaluable assistance with phosphorimager analysis of radioactivity.

\section{REFERENCES}

Bange, F. C., Collins, F. M. \& Jacobs, W. R., Jr (1999). Survival of mice infected with Mycobacterium smegmatis containing large DNA fragments from Mycobacterium tuberculosis. Tuber Lung Dis 79, 171-180.

Banu, S., Gordon, S. V., Palmer, S., Islam, M. R., Ahmed, S., Alam, K. M., Cole, S. T. \& Brosch, R. (2004). Genotypic analysis of Mycobacterium tuberculosis in Bangladesh and prevalence of the Beijing strain. J Clin Microbiol 42, 674-682.

Behr, M. A., Schroeder, B. G., Brinkman, J. N., Slayden, R. A. \& Barry, C. E., III (2000). A point mutation in the $m m a 3$ gene is responsible for impaired methoxymycolic acid production in Mycobacterium bovis BCG strains obtained after 1927. J Bacteriol 182, 3394-3399.

Besra, G. S. (1998). Preparation of cell-wall fractions from mycobacteria. In Mycobacteria Protocols, pp. 91-107. Edited by T. Parish \& N. G. Stoker. Totowa, NJ, USA: Humana Press.

Besra, G. S., Sievert, T., Lee, R. E., Slayden, R. A., Brennan, P. J. \& Takayama, K. (1994). Identification of the apparent carrier in mycolic acid synthesis. Proc Natl Acad Sci U S A 91, 12735-12739.

Bloch, K. (1977). Control mechanisms for fatty acid synthesis in Mycobacterium smegmatis. Adv Enzymol Relat Areas Mol Biol 45, 1-84. 
Brosch, R., Gordon, S. V., Billault, A., Garnier, T., Eiglmeier, K., Soravito, C., Barrell, B. G. \& Cole, S. T. (1998). Use of a Mycobacterium tuberculosis $\mathrm{H} 37 \mathrm{Rv}$ bacterial artificial chromosome library for genome mapping, sequencing, and comparative genomics. Infect Immun 66, 2221-2229.

Chen, J. M., Alexander, D. C., Behr, M. A. \& Liu, J. (2003). Mycobacterium bovis BCG vaccines exhibit defects in alanine and serine catabolism. Infect Immun 71, 708-716.

Converse, S. E., Mougous, J. D., Leavell, M. D., Leary, J. A., Bertozzi, C. R. \& Cox, J. S. (2003). MmpL8 is required for sulfolipid-1 biosynthesis and Mycobacterium tuberculosis virulence. Proc Natl Acad Sci U S A 100, 6121-6126.

Dobson, G., Minnikin, D. E., Minnikin, S. M., Parlett, J. H. \& Goodfellow, M. (1985). Systematic analysis of complex mycobacterial lipids. In Chemical Methods In Bacterial Systematics, pp. 237265. Edited by M. Goodfellow \& D. E. Minnikin. London: Academic Press.

Flick, P. K. \& Bloch, K. (1975). Reversible inhibition of the fatty acid synthetase complex from Mycobacterium smegmatis by palmitoylcoenzyme A. J Biol Chem 250, 3348-3351.

Gagneux, S., DeRiemer, K., Van, T., Kato-Maeda, M., de Jong, B. C., Narayanan, S., Nicol, M., Niemann, S., Kremer, K. \& other authors (2006). Variable host-pathogen compatibility in Mycobacterium tuberculosis. Proc Natl Acad Sci U S A 103, 2869-2873.

Garnier, T., Eiglmeier, K., Camus, J. C., Medina, N., Mansoor, H., Pryor, M., Duthoy, S., Grondin, S., Lacroix, C. \& other authors (2003). The complete genome sequence of Mycobacterium bovis. Proc Natl Acad Sci U S A 100, 7877-7882.

Glickman, M. S., Cox, J. S. \& Jacobs, W. R., Jr (2000). A novel mycolic acid cyclopropane synthetase is required for cording, persistence, and virulence of Mycobacterium tuberculosis. Mol Cell 5, 717-727.

Glickman, M. S., Cahill, S. M. \& Jacobs, W. R., Jr (2001). The Mycobacterium tuberculosis cmaA2 gene encodes a mycolic acid transcyclopropane synthetase. J Biol Chem 276, 2228-2233.

Goodfellow, M., Collins, M. D. \& Minnikin, D. E. (1976). Thin-layer chromatographic analysis of mycolic acid and other long-chain components in whole-organism methanolysates of coryneform and related taxa. J Gen Microbiol 96, 351-358.

Gould, T. A., van de Langemheen, H., Munoz-Elias, E. J., McKinney, J. D. \& Sacchettini, J. C. (2006). Dual role of isocitrate lyase 1 in the glyoxylate and methylcitrate cycles in Mycobacterium tuberculosis. Mol Microbiol 61, 940-947.

Guerin, C. (1980). The history of BCG. In BCG Vaccine: Tuberculosis-Cancer. Edited by S. Rosenthal. Littleton, MA, USA: PSG Publishing.

Gutierrez, M. C., Brisse, S., Brosch, R., Fabre, M., Omais, B., Marmiesse, M., Supply, P. \& Vincent, V. (2005). Ancient origin and gene mosaicism of the progenitor of Mycobacterium tuberculosis. PLoS Pathog 1, e5.

Henao-Tamayo, M., Junqueira-Kipnis, A. P., Ordway, D., GonzalesJuarrero, M., Stewart, G. R., Young, D. B., Wilkinson, R. J., Basaraba, R. J. \& Orme, I. M. (2007). A mutant of Mycobacterium tuberculosis lacking the $19-\mathrm{kDa}$ lipoprotein Rv3763 is highly attenuated in vivo but retains potent vaccinogenic properties. Vaccine 25, 7153-7159.

Iram, S. H. \& Cronan, J. E. (2005). Unexpected functional diversity among FadR fatty acid transcriptional regulatory proteins. $J$ Biol Chem 280, 32148-32156.

Jetten, M. S. \& Sinskey, A. J. (1995). Recent advances in the physiology and genetics of amino acid-producing bacteria. Crit Rev Biotechnol 15, 73-103.

Kamerbeek, J., Schouls, L., Kolk, A., van Agterveld, M., van Soolingen, D., Kuijper, S., Bunschoten, A., Molhuizen, H., Shaw, R. \& other authors
(1997). Simultaneous detection and strain differentiation of Mycobacterium tuberculosis for diagnosis and epidemiology. J Clin Microbiol 35, 907-914.

Keating, L. A., Wheeler, P. R., Mansoor, H., Inwald, J. K., Dale, J., Hewinson, R. G. \& Gordon, S. V. (2005). The pyruvate requirement of some members of the Mycobacterium tuberculosis complex is due to an inactive pyruvate kinase: implications for in vivo growth. Mol Microbiol 56, 163-174.

Marrakchi, H., Bardou, F., Laneelle, M.-A. \& Daffe, M. (2008). A comprehensive overview of mycolic acid structure and biosynthesis. In The Mycobacterial Cell Envelope, pp. 41-62. Edited by M. Daffé \& J.-M. Reyrat. Washington, DC: American Society for Microbiology.

McNeil, M., Daffé, M. \& Brennan, P. J. (1990). Evidence for the nature of the link between the arabinogalactan and peptidoglycan of mycobacterial cell walls. J Biol Chem 265, 18200-18206.

Minnikin, D. E., Kremer, L., Dover, L. G. \& Besra, G. S. (2002). The methyl-branched fortifications of Mycobacterium tuberculosis. Chem Biol 9, 545-553.

Mostowy, S., Inwald, J., Gordon, S., Martin, C., Warren, R., Kremer, K., Cousins, D. \& Behr, M. A. (2005). Revisiting the evolution of Mycobacterium bovis. J Bacteriol 187, 6386-6395.

Munoz-Elias, E. J., Upton, A. M., Cherian, J. \& McKinney, J. D. (2006). Role of the methylcitrate cycle in Mycobacterium tuberculosis metabolism, intracellular growth, and virulence. Mol Microbiol 60, 1109-1122.

Niederweis, M. (2003). Mycobacterial porins - new channel proteins in unique outer membranes. Mol Microbiol 49, 1167-1177.

Rainwater, D. L. \& Kolattukudy, P. E. (1982). Isolation and characterization of acyl coenzyme A carboxylases from Mycobacterium tuberculosis and Mycobacterium bovis, which produce multiple methyl-branched mycocerosic acids. J Bacteriol 151, 905911.

Reed, M. B., Domenech, P., Manca, C., Su, H., Barczak, A. K., Kreiswirth, B. N., Kaplan, G. \& Barry, C. E., III (2004). A glycolipid of hypervirulent tuberculosis strains that inhibits the innate immune response. Nature 431, 84-87.

Savvi, S., Warner, D. F., Kana, B. D., McKinney, J. D., Mizrahi, V. \& Dawes, S. S. (2008). Functional characterization of a vitamin B12dependent methylmalonyl pathway in Mycobacterium tuberculosis: implications for propionate metabolism during growth on fatty acids. J Bacteriol 190, 3886-3895.

Sinsimer, D., Huet, G., Manca, C., Tsenova, L., Koo, M. S., Kurepina, N., Kana, B., Mathema, B., Marras, S. A. \& other authors (2008). The phenolic glycolipid of Mycobacterium tuberculosis differentially modulates the early host cytokine response but does not in itself confer hypervirulence. Infect Immun 76, 3027-3036.

Smith, N. H., Dale, J., Inwald, J., Palmer, S., Gordon, S. V., Hewinson, R. G. \& Smith, J. M. (2003). The population structure of Mycobacterium bovis in Great Britain: clonal expansion. Proc Natl Acad Sci U S A 100, 15271-15275.

Smith, N. H., Gordon, S. V., de la Rua-Domenech, R., Clifton-Hadley, R. S. \& Hewinson, R. G. (2006). Bottlenecks and broomsticks: the molecular evolution of Mycobacterium bovis. Nat Rev Microbiol 4, 670-681.

van Aalten, D. M., DiRusso, C. C. \& Knudsen, J. (2001). The structural basis of acyl coenzyme A-dependent regulation of the transcription factor FadR. EMBO J 20, 2041-2050.

Wheeler, P. R. (2008). Analysis of lipid biosynthesis and location. In Mycobacteria Protocols, 2nd edn. Edited by T. Parish \& A. Brown. Totowa, NJ: Humana Press (in press). 
Wheeler, P. R. \& Anderson, P. M. (1996). Determination of the primary target for isoniazid in mycobacterial mycolic acid biosynthesis with Mycobacterium aurum $\mathrm{A}^{+}$. Biochem J 318, 451457.

Wheeler, P. R. \& Ratledge, C. (1988). Use of carbon sources for lipid biosynthesis in Mycobacterium leprae: a comparison with other pathogenic mycobacteria. J Gen Microbiol 134, 2111-2121.

Wheeler, P. R., Coldham, N. G., Keating, L., Gordon, S. V., Wooff, E. E., Parish, T. \& Hewinson, R. G. (2005). Functional demonstration of reverse transsulfuration in the Mycobacterium tuberculosis complex reveals that methionine is the preferred sulfur source for pathogenic mycobacteria. J Biol Chem 280, 8069-8078.

Winder, C. L., Gordon, S. V., Dale, J., Hewinson, R. G. \& Goodacre, R. (2006). Metabolic fingerprints of Mycobacterium bovis cluster with molecular type: implications for genotype-phenotype links. Microbiology 152, 2757-2765.

Edited by: G. R. Stewart 